\title{
Assessment of Cerebrovascular Reactivity during Resting State Breathing and Its Correlation with Cognitive Function in Hypertension
}

\author{
Ihab Hajjar ${ }^{a}$ Vasilis Marmerelis ${ }^{b}$ Dae C. Shin ${ }^{b}$ Helena Chui ${ }^{c}$ \\ a Division of Geriatrics and General Internal Medicine, Department of Medicine, Emory University, Atlanta, Ga., \\ Departments of ${ }^{b}$ Biomedical Engineering and ${ }^{\mathrm{C}}$ Neurology, University of Southern California, Los Angeles, Calif., USA
}

\section{Key Words \\ Hypertension · Cerebral blood flow · Trail-Making Test}

\begin{abstract}
Background: Hypertension is associated with cognitive deficits, particularly executive function, and decreased cerebral microvascular responsiveness to $\mathrm{CO}_{2}\left(\mathrm{CO}_{2}\right.$ vasoreactivity). The relation between $\mathrm{CO}_{2}$ vasoreactivity and executive function is not known. Protocols to assess $\mathrm{CO}_{2}$ vasoreactivity are cumbersome and require inhaling a $\mathrm{CO}_{2}$-enriched gas. We explored the ability to measure $\mathrm{CO}_{2}$ vasoreactivity using endtidal $\mathrm{CO}_{2}$ fluctuations during normal breathing and the association of this measure with cognitive function in hypertension. Methods: Executive function (Trail-Making Test parts $A / B)$, memory, attention and blood flow velocity (BFV) in the middle cerebral artery using transcranial Doppler were measured in hypertensive subjects who were tapered off their treatment for 3 weeks. BFV was measured while sitting and normally breathing for $5 \mathrm{~min}$, followed by breathing $5 \% \mathrm{CO}_{2}$ gas and hyperventilation for 2 min each. We calculated $\mathrm{CO}_{2}$ vasoreactivity as the rate of $\mathrm{BFV}$ change from hypoventilation to hyperventilation, and as a model-derived measure using the normal breathing data. The latter was derived using nonlinear principal dynamic modes (PDM), which modelled the dynamic effect of fluctuations in end-tidal $\mathrm{CO}_{2}$ and blood pressure upon BFV during normal room-air respiration. Multiple regression analyses were used to correlate cerebral hemodynamics with cognitive measures. Results: Data were
\end{abstract}

\section{KARGER}

E-Mail karger@karger.com

www.karger.com/ced collected from 41 individuals with hypertension (mean age 71 years, 24\% African Americans, 61\% women, off antihypertensive therapy). Lower $\mathrm{CO}_{2}$ vasoreactivity was associated with a worse executive function test score using both calculation methods: $p$ value using the hyper/hypoventilation data was 0.04 and from the PDM analysis was 0.009. PDM calculations showed a stronger correlation with executive function (0.41 vs. 0.21 using the hyper/hypoventilation data). There were no associations with memory or attention measures. There was a weak but statistically significant correlation between the two calculation methods of $\mathrm{CO}_{2}$ vasoreactivity $\left(R^{2}=14 \%, p=0.02\right)$. Conclusion: This study suggests that the decrease in $\mathrm{CO}_{2}$ vasoreactivity in hypertension is associated with lower executive function. This may offer new insight into the vascular underpinning of cognitive decline in hypertension. We demonstrate that calculating $\mathrm{CO}_{2}$ vasoreactivity is possible during normal breathing. If replicated in future studies, this may offer a more convenient clinical way to assess $\mathrm{CO}_{2}$ vasoreactivity in hypertension and cognitive disorders.

(c) 2014 S. Karger AG, Basel

\section{Introduction}

Hypertension leads to cognitive impairment and dementia [1]. Early on, hypertension is commonly associated with a decline in executive function that may predict future cognitive and functional loss [2,3]. It is suggested that this association is related to its effect on the microvas- 
culature of the brain. Hypertension is also associated with decreased responsiveness to $\mathrm{CO}_{2}[4]$, which may be an early marker of microvascular dysfunction and impaired endothelial health [5]. We therefore hypothesized that lower $\mathrm{CO}_{2}$ vasoreactivity in hypertension is associated with lower executive function. To our knowledge, no prior study has explored this association in hypertensive individuals.

In healthy individuals, cerebral blood flow is tightly regulated to meet the metabolic demands of the brain. The ability to maintain this metabolic demand can be assessed using transcranial Doppler. Two main indicators of cerebral hemodynamics have been commonly measured: (i) the ability to maintain cerebral blood flow velocity (BFV) during fluctuating perfusion pressure (autoregulation) and (ii) alterations in BFV due to arteriolar constriction or dilatation triggered by fluctuating end-tidal $\mathrm{CO}_{2}\left(\mathrm{CO}_{2}\right.$ vasoreactivity). Hypertension and aging are associated with decreased $\mathrm{CO}_{2}$ vasoreactivity but relatively preserved autoregulation [6-8]. Lower $\mathrm{CO}_{2}$ vasoreactivity is linked to an increased risk of vascular brain injury and death $[9,10]$.

Artificial changes in systemic blood pressure can be induced by standing. End-tidal $\mathrm{CO}_{2}$ can be induced by breath holding, breathing $\mathrm{CO}_{2}$-enriched air, or administering a dose of acetazolamide [11]. These maneuvers can be cumbersome. Furthermore, prior methods of calculating $\mathrm{CO}_{2}$ vasoreactivity used linear modeling and frequency-dependent linear transfer functions [12-15]. End-tidal $\mathrm{CO}_{2}$ fluctuates during normal breathing. However, the relation between end-tidal $\mathrm{CO}_{2}$ and BFV is nonlinear and there is low linear coherence at low frequencies [16]. Hence, the linear methods may not be very representative of the physiology of $\mathrm{CO}_{2}$ vasoreactivity during normal breathing. Deriving $\mathrm{CO}_{2}$ vasoreactivity measure from these fluctuations requires signal modelling methods that address these limitations (nonlinearity and low frequency).

In this study, we aimed at investigating the association between $\mathrm{BFV}$ autoregulation and $\mathrm{CO}_{2}$ vasoreactivity with cognitive function in hypertension. We derived the $\mathrm{CO}_{2}$ vasoreactivity using a novel modeling method developed by our coauthor from the resting respiratory fluctuations of end-tidal $\mathrm{CO}_{2}$, as well as using conventional methods during breathing $\mathrm{CO}_{2}$-enriched air and hyperventilation. We compared the two methods in their correlation with cognitive function.

\section{Materials and Methods}

\section{Study Description and Participants}

This is an analysis of the baseline data collected on the participants of a randomized clinical trial. The study design of the original clinical trial was described elsewhere [17]. Briefly, this was a 12-month double-blind randomized controlled clinical trial of candesartan, lisinopril or HCTZ. The data used in this analysis were collected at baseline. Inclusion criteria were: 60 years or older; hypertension (systolic blood pressure, SBP, $140 \mathrm{~mm} \mathrm{Hg}$ or greater, or diastolic blood pressure, DBP, $90 \mathrm{~mm} \mathrm{Hg}$ or greater, or receiving antihypertensive medications), and executive dysfunction based on a score less than 10 on the executive Clock-Drawing Test (CLOX1) [18]. To exclude those with possible dementia we did not enroll those with a Mini-Mental State Examination $<20$ or those with a clinical diagnosis of Alzheimer's disease or other dementias. Exclusion criteria included: intolerance to the study medications; SBP $>200$ or DBP $>110 \mathrm{~mm} \mathrm{Hg}$; elevated serum creatinine $(>2.0 \mathrm{mg} / \mathrm{dl})$ or serum potassium $(5.3 \mathrm{mEq} / \mathrm{dl})$ at baseline; receiving more than two antihypertensive medications; congestive heart failure; diabetes mellitus; stroke, and inability to perform the study procedures or unwilling to stop currently used antihypertensive medications. The subjects were recruited from the greater Boston area.

Participants who were receiving antihypertensive medications were instructed to stop their current therapy. All participants were provided with a portable automated blood pressure monitor and study personnel trained each participant on its use. All participants received written instructions on the tapering and discontinuation of antihypertensive medications and description of symptoms associated with possible adverse events. They were asked to measure blood pressure twice a day (morning and before sleep) and record them into a diary. Contact by the study personnel was twice weekly for the review of blood pressures. The timeline for medication was a decrease by $25-50 \%$ during week 1, 50-75\% during week 2 and $100 \%$ for week 3 . Baseline measurements were performed after 3 weeks. Subjects with significant blood pressure elevations (SBP $>180 / 100 \mathrm{~mm} \mathrm{Hg}$ ) for at least two consecutive measurements or who developed hypertension-related symptoms were excluded (failed taper), were asked to resume their usual dose of antihypertensive medications, and were referred back to their primary care physician for chronic blood pressure management as usual. The Institutional Review Board of the Hebrew SeniorLife approved the study and all participants provided written informed consent. The study was also registered with ClinicalTrials.gov (NCT00605072).

Baseline measurements were made (when the patients were off antihypertensive medications). Neuropsychological assessments included the Trail-Making Test (TMT) parts A, B [19]. TMT is a benchmark test for executive function. We also calculated TMT part B minus part A (B-A) which adjusts the TMT for the motor speed and dexterity of the participant [20]. We also used the revised Hopkins Verbal Learning Test (HVLT), and the Digit Span Test (DST). The HVLT assesses both immediate and delayed recall and recognition abilities, whilst the DST measures attention.

\section{Cerebral Hemodynamic Measurements}

$\mathrm{BFV}$ was measured in the middle cerebral artery using transcranial Doppler ultrasonography $(2-\mathrm{MHz}$ probe placed over the temporal bone; MultiDop X4, DWL-Transcranial Doppler Systems Inc., Sterling, Va., USA). Heart rate and blood pressure were simultaneously measured using a continuous ECG recording and a Finometer ${ }^{\mathrm{TM}}$ (FMS, Finapress Measurement Systems, Arnhem, The Netherlands). End-tidal $\mathrm{CO}_{2}$ was measured using a $\mathrm{CO}_{2}$ analyzer (VacuMed, Ventura, Calif., USA) attached to a nasal cannula. Measurements were obtained under the following conditions: (1) at rest while sitting for 5 min with normal breathing of 


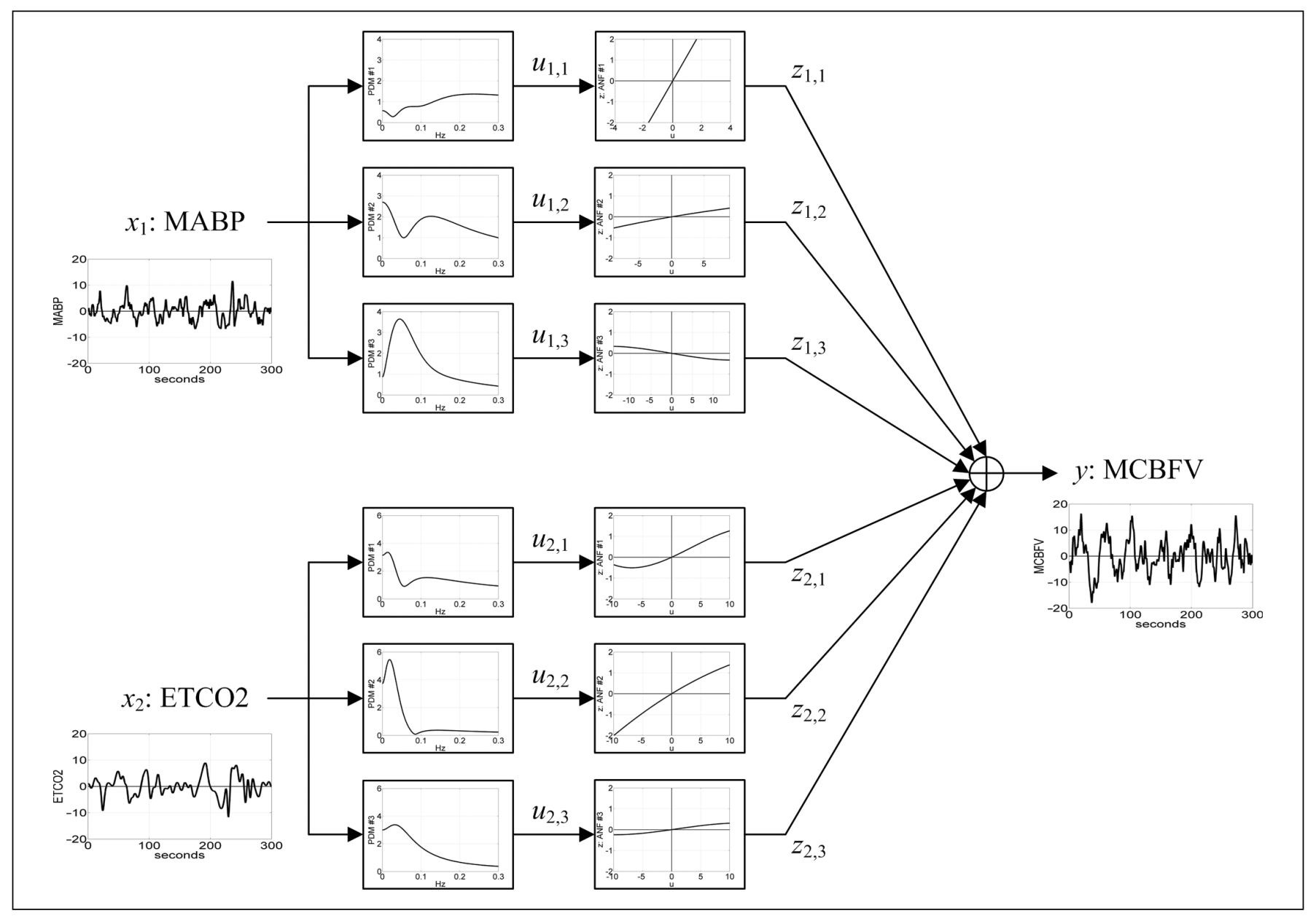

Fig. 1. Block diagram of the PDM-based model of cerebral hemodynamics with the output $y(t)$ being the beat-to-beat mean cerebral BFV (MCBFV) and two inputs: $\mathrm{x}_{1}(\mathrm{t})$ the mean arterial blood pressure $(\mathrm{MABP})$ and $\mathrm{x}_{2}(\mathrm{t})$ the end-tidal $\mathrm{CO}_{2}(\mathrm{ETCO} 2)$. There are three PDMs for each of the two inputs shown in the diagram in the frequency domain (transfer functions), which are common for all subjects and are estimated from the data. The output $\mathrm{u}_{\mathrm{j}, \mathrm{m}(\mathrm{t})}$ of the

room air; (2) during standing for $1 \mathrm{~min}$; (3) during breathing $8 \%$ $\mathrm{CO}_{2}$ air for $2 \mathrm{~min}$, and (4) during hyperventilating for $2 \mathrm{~min}$. Beatto-beat values of $\mathrm{BFV}$, blood pressure and end-tidal $\mathrm{CO}_{2}$ were computed offline from these continuous measurements using the recorded R-R intervals of the ECG.

\section{Calculation of Cerebral Hemodynamics Indices}

The obtained beat-to-beat time-series data were analyzed offline using Matlab (Mathworks, Natik, Mass., USA). We used the data that were measured after being off all antihypertensive medications.

\section{Autoregulation}

Using the data obtained at rest and during the 1-min stand, we calculated an index of autoregulation as the change in cerebrovascular resistance (CVR) denoted as delta-CVR. CVR is calculated as the ratio of mean arterial pressure divided by BFV. $\mathrm{j}^{\text {th }} \mathrm{PDM}$ for the $\mathrm{m}^{\text {th }}$ input is the convolution of the PDM with the respective input (linear filter operation). The associated nonlinear function (ANF) following each PDM is a cubic polynomial representing the system nonlinearities for the respective PDM and it is estimated from the data of each subject (subject specific). The output $\mathrm{y}(\mathrm{t})$ is the sum of all ANF outputs $\left(\mathrm{z}_{\mathrm{j}, \mathrm{m}}\right)$ and a constant offset value $\mathrm{c}_{0}$.

$\mathrm{CO}_{2}$ Vasoreactivity

This was calculated as the rate or slope of the change in BFV versus end-tidal $\mathrm{CO}_{2}$ during breathing enriched $\mathrm{CO}_{2}$ and hyperventilation, with ranges from hypocapnia to hypercapnia.

\section{Principal Dynamic Modes}

We also used the method of principal dynamic modes (PDM) to calculate $\mathrm{CO}_{2}$ vasoreactivity from 5-min normal breathing data [21, 22]. PDM constitute an efficient basis of reference functions for the representation of the system's kernels that can describe the dynamic relationship between input variables (e.g. blood pressure and endtidal $\mathrm{CO}_{2}$ ) and output variables (e.g. BFV) in a reliable and compact manner. A diagrammatic representation of the PDM modelling approach is shown in figure 1. Using the PDM-based models that have predictive capability, we can compute appropriate 'physiomarkers' of cerebral autoregulation and vasoreactivity for each subject by simu- 
lating the resulting $\mathrm{BFV}$ changes for given changes of blood pressure and $\mathrm{CO}_{2}$, respectively, during normal breathing. These physiomarkers were computed as the time-average of the PDM-based modelpredicted responses (beat-to-beat BFV) to step changes of end-tidal $\mathrm{CO}_{2}$ for vasoreactivity or arterial blood pressure for cerebral autoregulation relative to the respective baseline value over $30 \mathrm{~s}$. The magnitude of the step change was set equal to one standard deviation of the actual $\mathrm{CO}_{2}$ or pressure data recorded in each subject (i.e. adjusted to the observed operating range of each subject) and was centered around the baseline $\mathrm{CO}_{2}$ or pressure value (i.e. the average of the respective recorded data). This magnitude varied between 2.4 and $4.7 \mathrm{~mm} \mathrm{Hg}$ for the pressure changes, and between 1.6 and 5.9 $\mathrm{mm} \mathrm{Hg}$ for the $\mathrm{CO}_{2}$ changes in this set of patients. In order to obtain credible PDM-based model estimates we need at least 3 min of reliable beat-to-beat data [23]. We extracted the 3-min data from the 5 -min data recorded at rest. Since $\mathrm{CO}_{2}$ may increase blood pressure, these model-based indices were computed under closed-loop conditions where such reciprocal effects (i.e. of blood flow and $\mathrm{CO}_{2}$ changes upon blood pressure) are also taken into account [24]. A full mathematical description of this method was described elsewhere [25]. The PDM-based models are not ad hoc models, but rather they are canonical representations of cerebral hemodynamics, yielding indices of physiological meaning. Specifically, the resulting PDMbased $\mathrm{CO}_{2}$ vasoreactivity index represents the time average of BFV increase over $30 \mathrm{~s}$ per unit of imposed percent change in end-tidal $\mathrm{CO}_{2}(\mathrm{~cm} / \mathrm{s} / \% \mathrm{~mm} \mathrm{Hg})$. Likewise, the resulting model-based cerebral autoregulation index represents the time-average of cerebral flow velocity increase (in the middle cerebral artery) over $30 \mathrm{~s}$ per unit of imposed arterial pressure change $(\mathrm{cm} / \mathrm{s} / \mathrm{mm} \mathrm{Hg})$.

\section{Statistical Analysis}

Associations of the 'traditional' and PDM-derived physiomarkers of cerebral hemodynamics with cognitive function were assessed using linear regression models: the cognitive score as the dependent variable and the physiomarkers as the independent variable. TMT score distributions were skewed and hence we used natural logarithmic transformations. All regression models were adjusted for age and SBP. To assess the degree of association between PDM-derived indices or the 'traditional' linear physiomarkers with cognitive function we calculated two measures: the Pearson correlation and the partial $\mathrm{R}^{2}$ (as a measure of the proportion of the explained variation in the cognitive test score by the physiomarkers) calculated form the multiple regression models [26]. The SAS statistical package (SAS, Carey, N.C., USA) was used for these analyses.

\section{Results}

Of the 63 eligible participants, 53 tapered their antihypertensive medications and were assessed at baseline. Of those, 47 (89\%) had successful insonation of the middle cerebral artery. PDM-based indices were obtainable from 41 subjects because 6 had temporary displacement of the nasal cannula that reduced the available continuous timeseries data record to less than $3 \mathrm{~min}$, which renders the indices inaccurate.

Vasoreactivity and Executive Function in Hypertension
Table 1. Socio-demographic, clinical, neuropsychiatric and cerebral hemodynamic characteristics of the final sample available for this analysis

\begin{tabular}{lc}
$\mathrm{n}$ & 41 \\
Age, years & $71(7)$ \\
Women, \% & $25(61)$ \\
African American, \% & $10(24)$ \\
White, \% & $29(70)$ \\
High school education or lower, \% & $8(20)$ \\
College education or higher, \% & $33(80)$ \\
${\text { Body mass index, } \mathrm{kg} / \mathrm{m}^{2}}^{2}$ & $28.78(6.09)$ \\
\hline
\end{tabular}

\begin{tabular}{lr}
\hline Baseline cognitive function & \\
Mini-Mental State Examination & $26(2)$ \\
CLOX1 & $8(2)$
\end{tabular}

Blood pressure and heart rate when sitting

$\mathrm{SBP}, \mathrm{mm} \mathrm{Hg} \quad 151$ (16)

$\mathrm{DBP}, \mathrm{mm} \mathrm{Hg} \quad 83(9)$

Heart rate, beats/min $65(9)$

Cognitive function

TMT part A, s $37(12)$

TMT part B, s $100(45)$

TMT part B-A, s 63 (38)

HVLT recall $8(3)$

HVLT recognition $23(2)$

DST forward $6(1)$

DST backward $4(2)$

Cerebral hemodynamics

Sitting blood flow velocity, $\mathrm{cm} / \mathrm{s}$

$28.34(6.54)$

$\mathrm{CO}_{2}$ reactivity (slope of blood flow velocity

versus end-tidal $\mathrm{CO}_{2}$ )

CVR change (sit-to-stand)

$0.51(0.26)$

$-0.45(0.52)$

Principal dynamic modes indices

Pressure regulation index

$-0.13(0.80)$

$\mathrm{CO}_{2}$ vasoreactivity index

$0.41(1.10)$

Data for each measure are either mean with standard deviation in parentheses, or number with frequency in parentheses.

Basic clinical and hemodynamic characteristics are provided in table 1 . There was a weak but significant correlation between the $\mathrm{CO}_{2}$ vasoreactivity measures using the two calculation methods: $\mathrm{R}^{2}$ for the correlation was $14 \%, \mathrm{p}=0.02$. This correlation was stronger during the hypoventilation phase of the hyper/hypoventilation protocol $\left(\mathrm{R}^{2}\right.$ was $21 \%$ for hypoventilation and $1 \%$ for hyperventilation).

After adjusting for age and SBP, lower $\mathrm{CO}_{2}$ vasoreactivity, calculated as the rate (slope) of change in $\mathrm{BFV}$ versus the change in end-tidal $\mathrm{CO}_{2}$ during the hypo/hyperventilation procedure, was associated with worse performance on executive function testing [TMT part $\mathrm{B}(\mathrm{p}=0.009)$ and $\mathrm{B}$-A ( $\mathrm{p}=0.02)$ ] but not the other cognitive domains, as described in table 2. Also, a lower PDM-based index of 
Table 2. Association between cognitive function and resting $\mathrm{BFV}$, conventional $\mathrm{CO}_{2}$ vasoreactivity and autoregulation indices

\begin{tabular}{|c|c|c|c|c|c|c|}
\hline & \multicolumn{2}{|l|}{ Resting BFV } & \multicolumn{2}{|c|}{$\mathrm{CO}_{2}$ vasoreactivity } & \multicolumn{2}{|c|}{ CVR change (sit-to-stand) } \\
\hline & $\beta$ & $\mathrm{p}$ & $\beta$ & $\mathrm{p}$ & $\beta$ & $\mathrm{p}$ \\
\hline TMT part A & $0.001(0.01)$ & 0.69 & $-0.4(0.2)$ & 0.03 & $0.2(0.1)$ & 0.05 \\
\hline TMT part B & $-0.01(0.01)$ & 0.31 & $-0.7(0.3)$ & 0.009 & $-0.02(0.14)$ & 0.91 \\
\hline TMT part B-A & $-0.02(0.02)$ & 0.34 & $-0.9(0.4)$ & 0.02 & $-0.13(0.21)$ & 0.53 \\
\hline HVLT recall & $0.11(0.07)$ & 0.13 & $0.4(1.8)$ & 0.83 & $0.95(0.9)$ & 0.3 \\
\hline HVLT recognition & $0.003(0.05)$ & 0.95 & $0.07(1.1)$ & 0.95 & $0.21(0.59)$ & 0.73 \\
\hline DST forward & $-0.05(0.04)$ & 0.19 & $-0.5(0.9)$ & 0.56 & $-0.19(0.47)$ & 0.69 \\
\hline DST backwards & $0.2(0.22)$ & 0.38 & $0.5(1)$ & 0.61 & $0.3(0.54)$ & 0.59 \\
\hline
\end{tabular}

Values in parentheses are standard error. Slope $(\beta)$, standard errors and p values were obtained from the regression models adjusted for age and SBP. TMT (A, B and B-A) were transformed using a logarithmic transformation due to their skewed distribution.

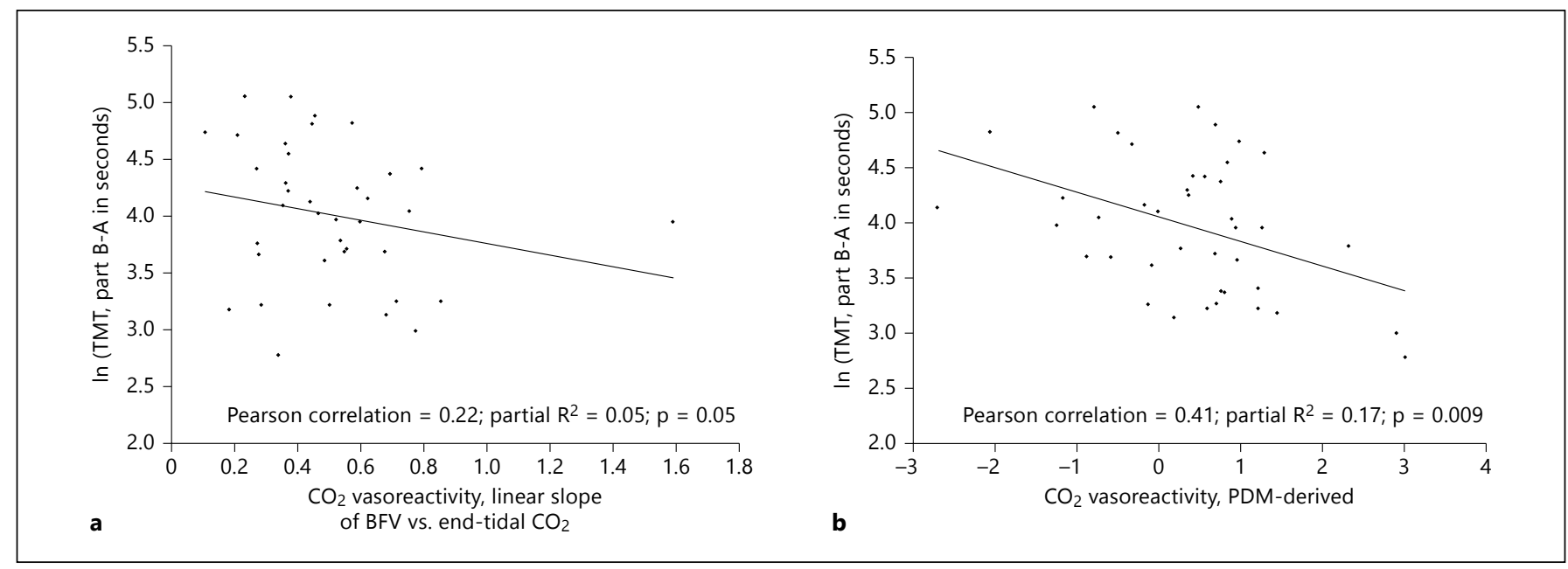

Fig. 2. Scatter-plot and regression association between TMT part $\mathrm{B}-\mathrm{A}$ included as the natural logarithm of TMT score and linear measure of $\mathrm{CO}_{2}$ vasoreactivity $\left(\mathrm{cm} / \mathrm{s} / \mathrm{mm} \mathrm{Hg}\right.$ end-tidal $\left.\mathrm{CO}_{2} ; \mathbf{a}\right)$

$\mathrm{CO}_{2}$ reactivity was associated with worse performances on TMT part B $(\mathrm{p}=0.05)$ and TMT part B-A $(\mathrm{p}=0.009)$.

Measures of association strength between executive function and $\mathrm{CO}_{2}$ vasoreactivity were higher for the $\mathrm{PDM}$-derived index of $\mathrm{CO}_{2}$ vasoreactivity compared to the traditional measure: the Pearson correlation of TMT part $\mathrm{B}$ with the linear measure of $\mathrm{CO}_{2}$ reactivity was 0.26 and with the PDM-derived index was 0.32 . The partial $\mathrm{R}^{2}$ was 0.07 for the traditional measure and 0.10 for the PDM-derived index. For TMT part B-A, the Pearson correlations were 0.22 for the traditional measure and 0.41 for the PDM-derived index. The partial $\mathrm{R}^{2}$ was 0.05 for the traditional versus 0.17 for the PDM-derived index. Illustrative scatter-plots with a fitted regression line of and PDM-derived measure of $\mathrm{CO}_{2}$ vasoreactivity (in $\mathrm{cm} / \mathrm{s} / \% \mathrm{CO}_{2}$ change; b). Partial $\mathrm{R}^{2}$ and $\mathrm{p}$ values were obtained from the multiple regression models adjusting for age and mean SBP.
TMT part B-A data versus the $\mathrm{CO}_{2}$-reactivity indices are shown in figure 2.

Sitting BFV values and autoregulation (delta-CVR or PDM derived) were not related to executive function, as shown in table 3. There was no association between any cerebral hemodynamic measure and the scores on the HVLT or the forward and backward DST.

\section{Discussion}

Our main findings are that lower $\mathrm{CO}_{2}$ vasoreactivity may be associated with worse executive function in hypertensive older adults, and calculating $\mathrm{CO}_{2}$ vasoreactiv- 
Table 3. Association between cognitive function and PDM-derived indices of cerebrovascular reactivity and pressure autoregulation

\begin{tabular}{|c|c|c|c|c|}
\hline & \multicolumn{2}{|c|}{$\mathrm{CO}_{2}$ vasoreactivity } & \multicolumn{2}{|c|}{ Pressure autoregulation } \\
\hline & $\beta$ & $\mathrm{p}$ & $\beta$ & $\mathrm{p}$ \\
\hline TMT part A & $0.02(0.04)$ & 0.63 & $0.09(0.06)$ & 0.15 \\
\hline TMT part B & $-0.11(0.06)$ & 0.05 & $0.04(0.08)$ & 0.68 \\
\hline TMT part B-A & $-0.22(0.08)$ & 0.01 & $0.01(0.13)$ & 0.95 \\
\hline HVLT recall & $0.4(0.38)$ & 0.3 & $0.04(0.54)$ & 0.94 \\
\hline HVLT recognition & $0.35(0.24)$ & 0.16 & $-0.22(0.35)$ & 0.53 \\
\hline DST forward & $-0.16(0.2)$ & 0.42 & $-0.42(0.27)$ & 0.13 \\
\hline DST backwards & $0.2(0.22)$ & 0.38 & $-0.16(0.32)$ & 0.63 \\
\hline
\end{tabular}

Values in parentheses are standard error. Slope $(\beta)$, standard errors and $\mathrm{p}$ values were obtained from the regression models adjusted for age and SBP. TMT (A, B and B-A) were transformed using a logarithmic transformation due to their skewed distribution.

ity using a nonlinear modelling technique from resting measurements of BFV and end-tidal $\mathrm{CO}_{2}$ normal breathing fluctuations is at least as useful as using the protocol of hypercapnia to hypocapnia. Our preliminary data suggest that the measure calculated using the normal breathing data might even be a stronger marker for executive function deficit in hypertension.

Although the underlying mechanisms are unknown, hypertension may preferentially affect executive function even in the absence of memory deficits. Prior studies have shown that hypertension is associated with a decline in $\mathrm{CO}_{2}$ vasoreactivity [8]. This study extends this association to show that the lower the $\mathrm{CO}_{2}$ vasoreactivity, the worse the executive function performance is. This suggests that damage to the microvasculature function may explain the observed predilection of atrophy in the prefrontal lobe and executive dysfunction with hypertension [27]. $\mathrm{CO}_{2}$ vasoreactivity may be a marker of endothelialdependent dilatation and the ability to react to changes in $\mathrm{pH}$ in the arterioles $[28,29]$. Wide use of $\mathrm{CO}_{2}$ vasoreactivity has been limited by the need to induce wide alterations in end-tidal $\mathrm{CO}_{2}$ (e.g. hyperventilation or breath holding). In this study we provide reliable quantitative means to assess $\mathrm{CO}_{2}$ vasoreactivity using only restingstate data of $\mathrm{BFV}$, end-tidal $\mathrm{CO}_{2}$ and systemic blood pressure. The advanced mathematical modelling using PDM can be automated to provide rapid, online physiomarkers of potential clinical utility (e.g. the vasoreactivity and autoregulation indices proposed herein) while the patient is being clinically assessed or shortly after. The present

Vasoreactivity and Executive Function in Hypertension study provides a first step to making this approach clinically feasible.

Multiple prior mathematical models of cerebral blood flow regulation have been developed [30]. The PDM model-based indices in this study performed as well as or even better than the traditional $\mathrm{CO}_{2}$ vasoreactivity measures in explaining the variance in executive function performance. The relative ease of obtaining these measures, compared to a full hypercapnia/hypocapnia protocol, and their association with executive function, in contrast to memory or attention, provide further impetus to explore these measures in future research.

Hypertension is associated with decreased $\mathrm{CO}_{2}$ vasoreactivity and antihypertensive therapy has a significant effect on cerebral blood flow and, hence, our study may be more reflective of the underlying pathological changes that occur with hypertension. The relatively small sample size is a limitation of this study. Another limitation is the lack of neuroimaging to confirm the presence of microvascular disease, and the lack of measurement of intracranial pressure, which is an important factor in determining BFV. Despite these limitations, our data add critical information to the field that, in hypertension, lower $\mathrm{CO}_{2}$ vasoreactivity may be associated with lower executive function but not memory declines. It is a first and critical step in unravelling the complex relation between hypertension and cognition.

\section{Conclusion}

Our study suggests that lower $\mathrm{CO}_{2}$ vasoreactivity is associated with worse executive function. PDM-based modeling using $\mathrm{BFV}$ and end-tidal $\mathrm{CO}_{2}$ during normal breathing would decrease the time and inconvenience of measuring this important vascular marker. Future research is needed on the utility of this measure in hypertensive brain health and related clinical trials.

\section{Acknowledgements}

Dr. Hajjar and the AVEC trial are supported by grant $1 \mathrm{~K} 23$ AG030057 from the National Institute on Aging. This work was supported in part by the Biomedical Simulations Resource at the University of Southern California under NIH/NIBIB grant P41EB001978.

\section{Disclosure Statement}

None. 


\section{References}

$\checkmark 1$ Novak V, Hajjar I: The relationship between blood pressure and cognitive function. Nat Rev Cardiol 2010;7:686-698.

-2 Vicario A, Martinez CD, Baretto D, Diaz Casale A, Nicolosi L: Hypertension and cognitive decline: impact on executive function. J Clin Hypertens (Greenwich) 2005;7:598-604

-3 Liu-Ambrose T, Pang MY, Eng JJ: Executive function is independently associated with performances of balance and mobility in community-dwelling older adults after mild stroke: implications for falls prevention. Cerebrovasc Dis 2007;23:203-210.

-4 Hajjar I, Zhao P, Alsop D, Novak V: Hypertension and cerebral vasoreactivity: a continuous arterial spin labeling magnetic resonance imaging study. Hypertension 2010;56: 859-864.

5 Cohen RA: Hypertension and cerebral blood flow: implications for the development of vascular cognitive impairment in the elderly. Stroke 2007;38:1715-1717.

-6 Carey BJ, Eames PJ, Blake MJ, Panerai RB, Potter JF: Dynamic cerebral autoregulation is unaffected by aging. Stroke 2000;31:28952900.

7 Eames PJ, Blake MJ, Panerai RB, Potter JF: Cerebral autoregulation indices are unimpaired by hypertension in middle aged and older people. Am J Hypertens 2003;16:746753.

$>8$ Lipsitz LA, Mukai S, Hamner J, Gagnon M, Babikian V: Dynamic regulation of middle cerebral artery blood flow velocity in aging and hypertension. Stroke 2000;31:1897-1903.

-9 Portegies ML, de Bruijn RF, Hofman A, Koudstaal PJ, Ikram MA: Cerebral vasomotor reactivity and risk of mortality: the Rotterdam study. Stroke 2014;45:42-47.

10 Yonas H, Smith HA, Durham SR, Pentheny SL, Johnson DW: Increased stroke risk predicted by compromised cerebral blood flow reactivity. J Neurosurg 1993;79:483-489.
11 Totaro R, Marini C, Baldassarre M, Carolei A: Cerebrovascular reactivity evaluated by transcranial Doppler: reproducibility of different methods. Cerebrovasc Dis 1999;9:142-145.

12 Aoi M, Gremaud P, Tran HT, Novak V, Olufsen MS: Modeling cerebral blood flow and regulation. Conf Proc IEEE Eng Med Biol Soc 2009;2009:5470-5473.

13 Peterson EC, Wang Z, Britz G: Regulation of cerebral blood flow. Int J Vasc Med 2011; 2011:523-525.

14 Giller CA: The frequency-dependent behavior of cerebral autoregulation. Neurosurgery 1990;27:362-368.

15 Zhang R, Zuckerman JH, Giller CA, Levine $\mathrm{BD}$ : Transfer function analysis of dynamic cerebral autoregulation in humans. Am J Physiol 1998;274:H233-H241.

16 Mitsis GD, Ainslie PN, Poulin MJ, Robbins PA, Marmarelis VZ: Nonlinear modeling of the dynamic effects of arterial pressure and blood gas variations on cerebral blood flow in healthy humans. Adv Exp Med Biol 2004;551: 259-265.

17 Hajjar I, Hart M, Milberg W, Novak V, Lipsitz $\mathrm{L}$ : The rationale and design of the antihypertensives and vascular, endothelial, and cognitive function (AVEC) trial in elderly hypertensives with early cognitive impairment: role of the renin angiotensin system inhibition. BMC Geriatr 2009;9:48.

18 Royall DR, Cordes JA, Polk M: CLOX: an executive clock drawing task. J Neurol Neurosurg Psychiatry 1998;64:588-594.

19 Gordon NG: The trail making test in neuropsychological diagnosis. J Clin Psychol 1972; 28:167-169.

20 Corrigan JD, Hinkeldey NS: Relationships between parts A and B of the Trail Making Test. J Clin Psychol 1987;43:402-409.
21 Marmarelis VZ, Shin DC, Orme ME, Zhang R: Model-based quantification of cerebral hemodynamics as a physiomarker for Alzheimer's disease? Ann Biomed Eng 2013;41:22962317.

22 Marmarelis VZ, Shin DC, Orme ME, Zhang R: Closed-loop dynamic modeling of cerebral hemodynamics. Ann Biomed Eng 2013;41: 1029-1048

23 Mitsis GD, Poulin MJ, Robbins PA, Marmarelis VZ: Nonlinear modeling of the dynamic effects of arterial pressure and $\mathrm{CO}_{2}$ variations on cerebral blood flow in healthy humans. IEEE Trans Biomed Eng 2004;51: 1932-1943.

24 Garnham J, Panerai RB, Naylor AR, Evans DH: Cerebrovascular response to dynamic changes in $\mathrm{pCO}_{2}$. Cerebrovasc Dis 1999;9: 146-151.

25 Marmarelis VZ, Shin DC, Orme ME, Zhang R: Closed-loop dynamic modeling of cerebral hemodynamics. Ann Biomed Eng 2013;41: 1029-1048.

26 Guilford JP, Fruchter B: Fundamental Statistics in Psychology and Education, ed 6. New York, McGraw-Hill, 1978.

27 Raz N, Rodrigue KM, Acker JD: Hypertension and the brain: vulnerability of the prefrontal regions and executive functions. Behav Neurosci 2003;117:1169-1180.

28 Heistad DD, Mayhan WG, Coyle P, Baumbach GL: Impaired dilatation of cerebral arterioles in chronic hypertension. Blood Vessels 1990;27:258-262.

29 Mayhan WG, Faraci FM, Heistad DD: Impairment of endothelium-dependent responses of cerebral arterioles in chronic hypertension. Am J Physiol 1987;253:H1435-H1440.

30 Gao E, Young WL, Pile-Spellman J, Ornstein E, Ma Q: Mathematical considerations for modeling cerebral blood flow autoregulation to systemic arterial pressure. Am J Physiol 1998;274:H1023-H1031. 